\title{
Design of 57.5 MHz cw RFQ for medium energy heavy ion superconducting linac
}

\author{
P. N. Ostroumov and A. A. Kolomiets* \\ Physics Division, Argonne National Laboratory, 9700 South Cass Avenue, Argonne, Illinois 60439
}

\begin{abstract}
D. A. Kashinsky, S. A. Minaev, V. I. Pershin, T. E. Tretyakova, and S. G. Yaramishev
Institute of Theoretical and Experimental Physics, Moscow 117259, Russia
\end{abstract}

(Received 25 January 2002; published 3 June 2002)

\begin{abstract}
The nuclear science community considers the construction of the Rare Isotope Accelerator (RIA) facility as a top priority. The RIA includes a $1.4 \mathrm{GV}$ superconducting linac for production of $400 \mathrm{~kW} \mathrm{cW}$ heavy ion beams. The initial acceleration of heavy ions delivered from an electron cyclotron resonance ion source can be effectively performed by a $57.5 \mathrm{MHz} 4-\mathrm{m}$ long room temperature RFQ. The principal specifications of the RFQ are (i) formation of extremely low longitudinal emittance, (ii) stable operation over a wide range of voltage for acceleration of various ion species needed for RIA operation, and (iii) simultaneous acceleration of two-charge states of uranium ions. cw operation of an accelerating structure leads to a number of requirements for the resonators such as high shunt impedance, efficient water cooling of all parts of the resonant cavity, mechanical stability together with precise alignment, reliable rf contacts, a stable operating mode, and fine tuning of the resonant frequency during operation. To satisfy these requirements a new resonant structure has been developed. This paper discusses the beam dynamics and electrodynamics design of the RFQ cavity, as well as some aspects of the mechanical design of the low-frequency cw RFQ.
\end{abstract}

DOI: 10.1103/PhysRevSTAB.5.060101

PACS numbers: 29.17.+w, 29.27.-a, 41.75.-i

\section{INTRODUCTION}

The initial acceleration of heavy ion beams in the driver linac of the Rare Isotope Accelerator (RIA) facility [1] will be provided by room temperature RFQ operating at 57.5 MHz. The primary scope of the RFQ is the acceleration of low longitudinal emittance dual charge state uranium beams. Transverse emittance should remain unchanged and a high beam capture efficiency is required. In order to simplify the front end of a multibeam driver linac and accommodate different ion species from the electron cyclotron resonance ion source, the RFQ must operate at a wide range of power levels. The basic RFQ design specifications are listed in Table I. Several types of resonant structures have been analyzed in order to satisfy all specifications. As a result, we propose an original RFQ structure which combines the advantages of the four-vane and split-coaxial structures. Four-vane RFQs provide high shunt impedances while split-coaxial structures have extremely good mode separation.

\section{BEAM DYNAMICS DESIGN}

The shape of the RFQ vanes (or electrodes) was designed using the code DESRFQ [2]. In this code the accelerating cell is determined as the distance between electrode transverse cross sections that have exact quadrupole symmetry. These cross sections are equivalent to the accelerat-

\footnotetext{
*On leave from Institute of Theoretical and Experimental Physics, Moscow, Russia.
}

ing gap centers in a standard drift tube linac. In the code, the length $L_{c i}$ of the $i$ th cell, for a given modulation factor $m_{i}$, is calculated iteratively so that a reference particle exits a cell at a given synchronous phase $\varphi_{s i}$. An additional condition is required to calculate the RFQ parameters $m_{i}$ and $\varphi_{s i}$ in each cell at a given peak surface field. This condition is discussed below.

For each iteration, DESRFQ calculates electric field distributions in a cell using a modified version of the code POLE1 [3]. Eight coefficients of the Fourier-Bessel series describing the potential distribution in a cell for a given shape of the electrodes are calculated by

TABLE I. Initial requirements for RFQ design.

\begin{tabular}{|c|c|}
\hline Duty cycle & $100 \%$ \\
\hline Operating frequency & $57.5 \mathrm{MHz}$ \\
\hline Resonant cavity & $\begin{array}{l}\text { Based on the split-coaxial } \\
\text { structure }\end{array}$ \\
\hline Input particle velocity & $0.00507 c$ \\
\hline Output particle velocity & $\sim 0.02 c$ \\
\hline Design charge-to-mass ratio & $28.5 / 238$ \\
\hline $\begin{array}{l}\text { Charge-to-mass ratio of } \\
\text { accelerated ions }\end{array}$ & $28 / 238 ; 29 / 238$ \\
\hline Length of the RFQ vanes & $\sim 4 \mathrm{~m}$ \\
\hline Peak field at electrode surface & $\leq 1.25$ Kilpatrick units \\
\hline Normalized transverse emittance & $0.5 \pi \mathrm{mm} \mathrm{mrad}$ \\
\hline $\begin{array}{l}\text { Longitudinal emittance at the exit } \\
\text { of RFQ for } 99.9 \%\end{array}$ & \\
\hline of particles & $\leq 2 \pi \mathrm{keV} / \mathrm{u}$ nsec \\
\hline
\end{tabular}




$$
\begin{aligned}
U(r, \psi, z) & =-\frac{U_{l}}{2}\left[F_{0}(r, \psi)+\sum_{n=1}^{\infty} F_{2 n}(r, \psi) \sin (2 n k z)+\sum_{n=1}^{\infty} F_{2 n-1}(r, \psi) \sin (2 n-1) k z\right], \\
F_{0}(r, \psi) & =\sum_{m=0}^{\infty} B_{s}\left(\frac{r}{R_{0}}\right)^{2(2 m+1)} \cos [2(2 m+1) \psi], \\
F_{2 n}(r, \psi) & =\sum_{m=0}^{\infty} A_{2 n, 2 m+1} I_{2(2 m+1)}(2 n k r) \cos [2(2 m+1) \psi], \\
F_{2 n-1}(r, \psi) & =\sum_{m=0}^{\infty} A_{2 n-1,2 m} I_{4 m}[(2 n-1) k r] \cos (4 m \psi),
\end{aligned}
$$

where $F_{n}, B_{s}$, and $A_{n s}$ are the coefficients of the FourierBessel expansion, $k=2 \pi / \beta \lambda, \beta$ is the relative ion velocity, $\lambda$ is the wavelength of the rf field, and $R_{0}$ is the characteristic (or average) radius of the RFQ. The vane tip is a semicircle with radius $R_{e}=0.75 R_{0}$. The distance from the RFQ axis to the vane tip in each $i$ th cell is determined by a sinusoidal curve, function of the longitudinal coordinate $z$ :

$$
\begin{aligned}
& x_{i}=R_{0}\left(1+\frac{m_{i}-1}{m_{i}+1} \sin \left(\pi z / L_{c i}\right)\right), \\
& y_{i}=R_{0}\left(1-\frac{m_{i}-1}{m_{i}+1} \sin \left(\pi z / L_{c i}\right)\right) .
\end{aligned}
$$

DESRFQ calculates the coefficients of the Fourier-Bessel series (1) for a sinusoidal shape of the vane tips.

If the peak surface field is specified, the stability region of transverse oscillation in the RFQ can be calculated for a given characteristic radius $R_{0}$. Figure 1 shows the phase advance and normalized acceptance in the transverse planes as a function of $R_{0}$. The curves were calculated for $R_{e} / R_{0}=0.75$. Choosing $R_{0}=0.6 \mathrm{~mm}$, we can obtain a transverse acceptance that is 4 times larger than the expected beam emittance from the ion source and also provide sufficiently strong transverse focusing. For this value of $R_{0}$, the maximum value $\rho_{\max }$ of the modulus of the

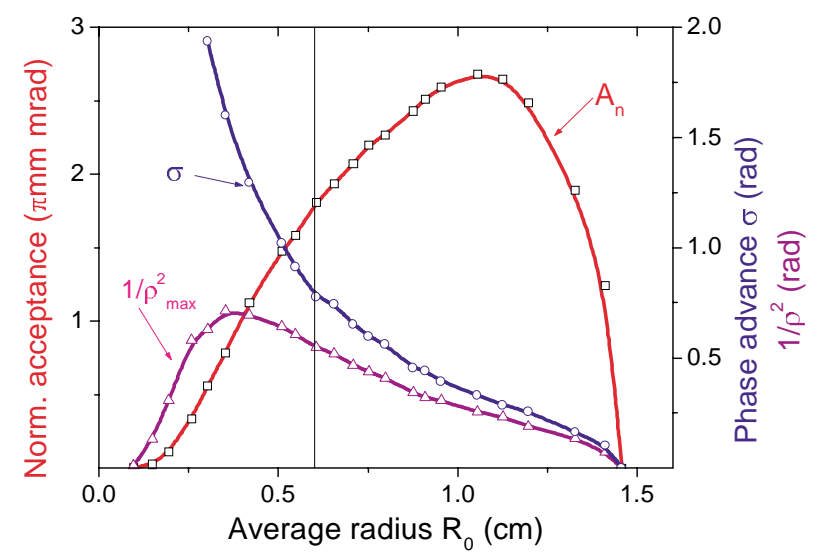

FIG. 1. (Color) Normalized acceptance $A_{n}$ (red line) and transverse phase advance $\sigma$ (blue line) calculated for a surface field $E_{\max }=140 \mathrm{kV} / \mathrm{cm}$. The magenta curve represents the parameter $1 / \rho_{\max }^{2}$, where $\rho_{\max }$ is the maximum value of the modulus of the Floquet function.
Floquet function $\rho(t)$ is small enough and the beam envelope $r_{\max }=\sqrt{\lambda \varepsilon_{n}} \rho_{\max }$ is about $2.3 \mathrm{~mm}$. As a consequence, the particles move within the linear region of the focusing fields. The matched beam parameters at the RFQ entrance were determined by an inverse transformation of $\rho(t)$ and $\frac{d \rho(t)}{d t}$ through six cell matching sections. In this case, the distance from the axis to the vane tip is given by

$$
x(z)=R_{0} \frac{1}{\sin \left(\pi \frac{z+0.5 L_{c 0}}{13 L_{c 0}}\right)}, \quad 0 \leq z \leq 6 L_{c 0},
$$

where $L_{c 0}$ is the length of the first RFQ cell.

As was discussed in our previous work [4], a multiharmonic buncher must be used upstream of the RFQ to produce the lowest possible longitudinal emittance of two-charge-state beams. The ion beam distribution in the longitudinal phase space at the RFQ entrance is determined by a four-harmonic buncher and is shown in Fig. 2. The central part of the distribution contains more than $80 \%$ of the particles within a phase width equal to $\pm 25^{\circ}$. The main goal of the beam dynamics design is to accept the core of the initial distribution and reliably eliminate halo particles from the acceleration process. This can be done if the acceleration starts with a small separatrix whose length is kept constant along the RFQ. The condition

$$
\frac{A_{10} \sin \varphi_{s}}{\beta^{2}}=\mathrm{const}
$$

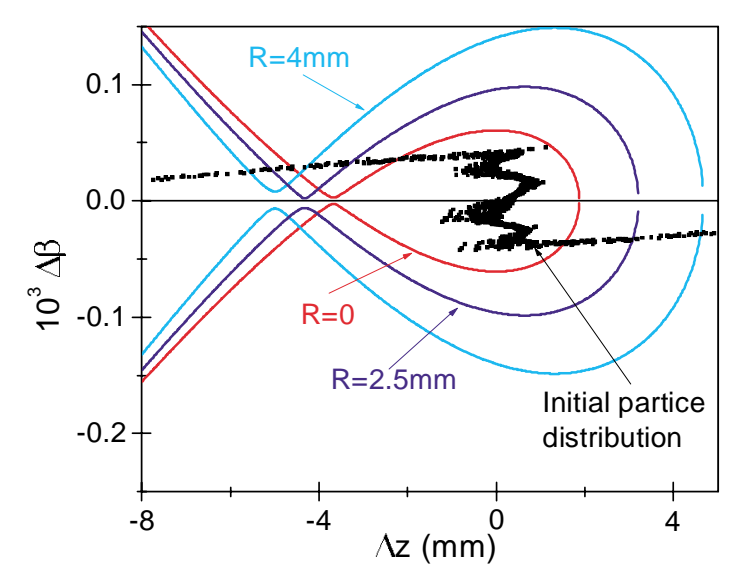

FIG. 2. (Color) Initial particle distribution and separatrices of the longitudinal motion calculated for different amplitudes of transverse oscillations at the injection velocity $\beta=0.00507$. 
ensures that all particle trajectories in phase space are conserved in the linear approximation. $A_{10}$ is the coefficient of the series (1). However, the situation is more compli- cated due to the strong coupling of the longitudinal and transverse motions in the front end of the RFQ, inherent in heavy ion RFQs. The Hamiltonian for longitudinal motion in an RFQ can be expressed as [5]

$$
H(\Delta z, \Delta \beta)=\frac{c \Delta \beta^{2}}{2}+\frac{q e U_{0} T}{\pi A W_{e}}\left[k \Delta z \cos \varphi_{s}-I_{0}^{2}\left(\frac{k R}{2}\right) \sin \left(k \Delta z-\varphi_{s}\right)\right],
$$

where $\Delta z, \Delta p$ are particle coordinates with respect to the reference particle in phase space, $q e$ is the ion charge, $W_{e}=m_{e} c^{2}, m_{e}$ is the atomic unit mass, $A$ is the mass number, $c$ is the speed of light, $U_{0}$ is the vane-to-vane voltage, $R$ is the average amplitude of transverse oscillations, and $I_{0}$ is the modified Bessel function. In the front end of the RFQ the term $I_{0}(k R / 2)$ is not small and the Hamiltonian strongly depends on amplitude of transverse oscillations. Figure 2 shows the separatrices calculated for the initial value of the synchronous particle velocity $\beta=0.00507$ for different amplitudes of the transverse oscillations. The initial values of $T$ and $\varphi_{s}$ can be chosen to accept the central dense area of the initial distribution into the separatrix corresponding to $R=0$. However, there are particles with large transverse amplitudes. These particles are captured for acceleration and have large longitudinal amplitudes. Obviously, they form a halo in the longitudinal phase space. As expected, the total longitudinal emittance can significantly exceed the emittance of the central part containing $\sim 80 \%-85 \%$ of the accelerated particles. An obvious way to weaken the coupling between the transverse and longitudinal motions is to increase the injection energy or the rf wavelength. However, increasing these parameters will have an impact on the construction cost of the RFQ.

To avoid resonant conditions between the frequency of transverse and longitudinal oscillations, the following condition must be satisfied in the RFQ [5]:

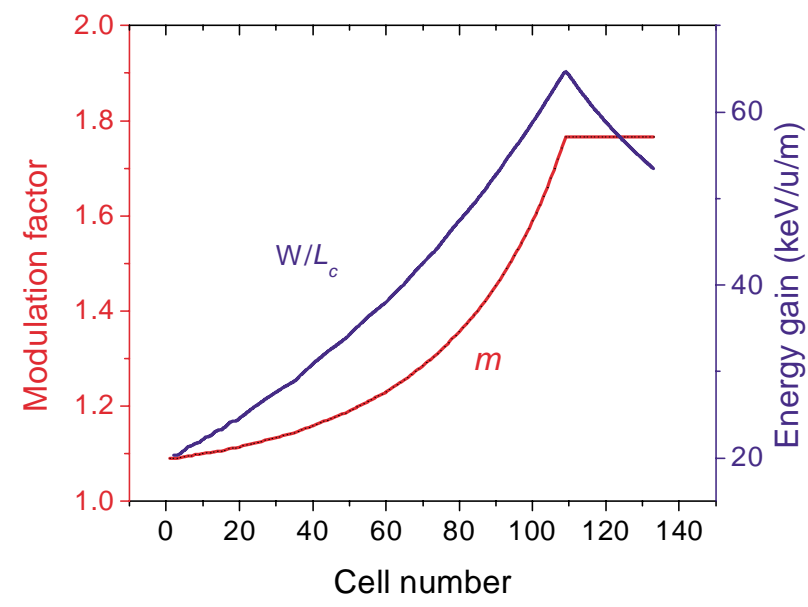

FIG. 3. (Color) Modulation factor $m$ and energy gain $W / L_{c}$ along the RFQ.

$$
\frac{\Omega_{l}}{\Omega_{r}}<\frac{1}{I_{0}\left(\frac{k a}{2}\right)},
$$

where $\Omega_{l}$ and $\Omega_{r}$ are the frequencies of small longitudinal and transverse oscillations, respectively, and $a$ is the RFQ aperture. In our design this condition is fulfilled along the whole RFQ.

TABLE II. Main RFQ parameters.

\begin{tabular}{lc}
\hline \hline Average radius $R_{0}$ & $0.6 \mathrm{~cm}$ \\
Vane tip radius $R_{e}$ & $0.45 \mathrm{~cm}$ \\
Vane-to-vane voltage $U_{0}$ & $68.5 \mathrm{kV}$ \\
Maximum field on the vane & $140 \mathrm{kV} / \mathrm{cm}$ \\
$\quad$ surface $E_{\max }$ & $199 \mathrm{keV} / \mathrm{u}$ \\
Output beam energy & $-25^{\circ}$ \\
Synchronous phase $\varphi_{s}$ & $1.09-1.765$ \\
Modulation factor & $0.43 \mathrm{~cm}$ \\
Aperture radius & \\
Phase advance of transverse & $44^{\circ}-41^{\circ}$ \\
$\quad$ oscillations $\sigma_{0}$ & \\
Normalized transverse frequency & 0.123 \\
$\quad \Omega_{r} / \omega, \omega=2 \pi c / \lambda$ & \\
Normalized longitudinal frequency & \\
$\quad \Omega_{l} / \omega$ & 0.04 \\
Normalized transverse acceptance & $1.8 \pi \mathrm{mm} \mathrm{mrad}$ \\
$\quad \Omega_{l} / \Omega_{r}$ & 0.326 \\
Vane length & $392 \mathrm{~cm}$ \\
\hline \hline
\end{tabular}

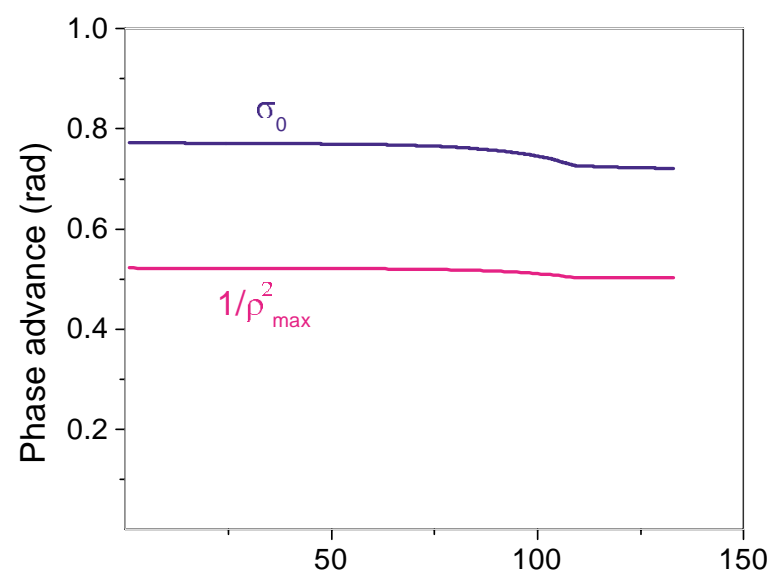

Cell number

FIG. 4. (Color) Phase advance $\sigma_{0}$ and minimum normalized transverse frequency $1 / \rho_{\max }^{2}$ along the RFQ. 
Since the external multiharmonic buncher produces short bunches at the RFQ entrance, the synchronous phase of the RFQ may be kept constant along the structure. The initial modulation was chosen so as to minimize the amplitude of energy oscillations. Next, the modulation factor varies according to relation (2) along the structure, up to the point where $\beta=0.017$ (Fig. 3). Downstream of this point the modulation factor is kept constant in order to maintain a high transverse phase advance.

The final parameters of the RFQ are presented in Table II and Figs. 3 and 4. The smooth behavior of transverse parameters $\sigma_{0}$ and $1 / \rho_{\max }$ as a function of the cell number guarantees matched motion of the particles along the RFQ.

\section{BEAM DYNAMICS SIMULATIONS}

The multiparticle code DYNAMION [6] was used for beam dynamics simulations in the RFQ. The electric field acting on each particle is calculated using coefficients of the series (1). The initial particle distribution, shown in Fig. 2,

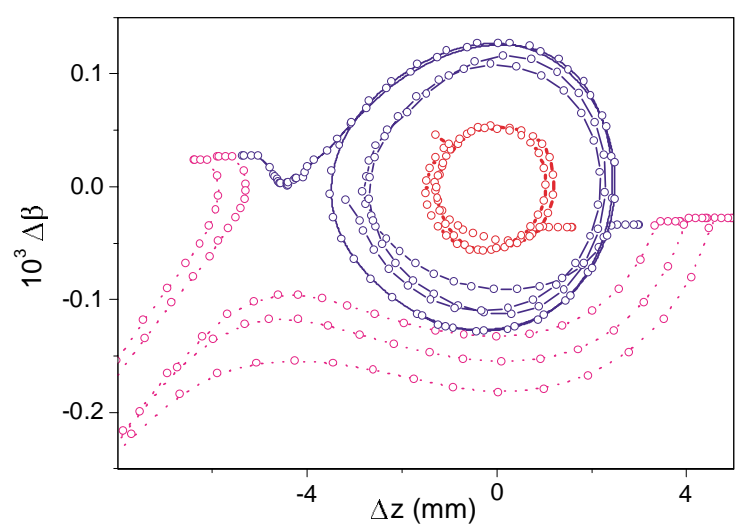

FIG. 5. (Color) Particle trajectories in the longitudinal phase space. The solid curves represent central trajectories and the dotted curves represent peripheral particles trajectories.

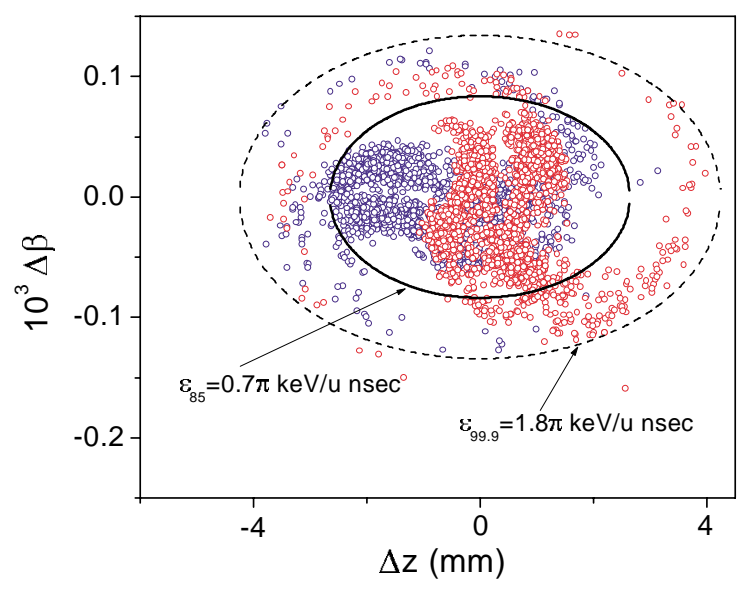

FIG. 6. (Color) Longitudinal phase space plots of particles exiting the RFQ. was obtained from three-dimensional beam dynamics simulations in the section of low energy beam transport containing the multiharmonic buncher. Figure 5 shows trajectories of some particles with different initial phases, plotted in the same coordinate system as in Fig. 2. Figure 5 presents particle trajectories for stable (solid curves) and unstable (dotted curves) motion. One can see that some particles with longitudinal amplitudes oscillating even larger than the separatrix corresponding to a particle moving in the RFQ axis are still captured. The fact that the trajectories are concentric confirms that the longitudinal motion is adiabatic during acceleration, as imposed in condition (2).

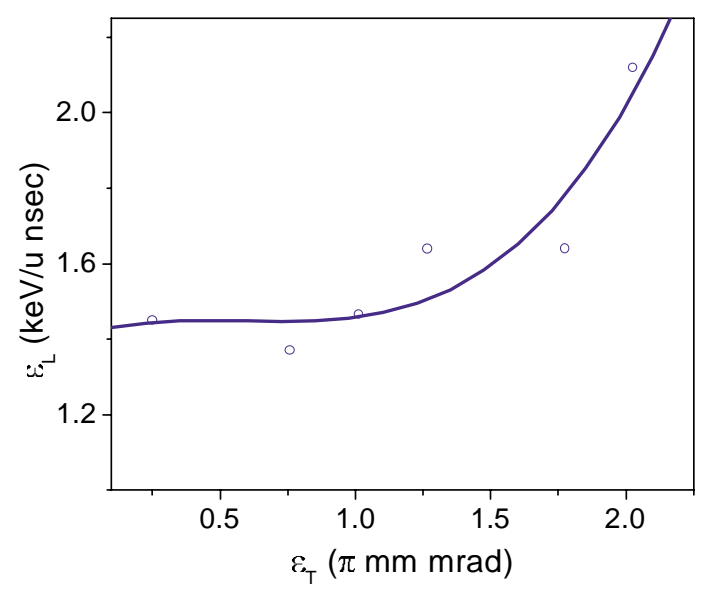

FIG. 7. (Color) Longitudinal emittance of $99.9 \%$ of the total accelerated particles $\varepsilon_{L}$ in the RFQ as a function of input transverse normalized emittance $\varepsilon_{n T}$.
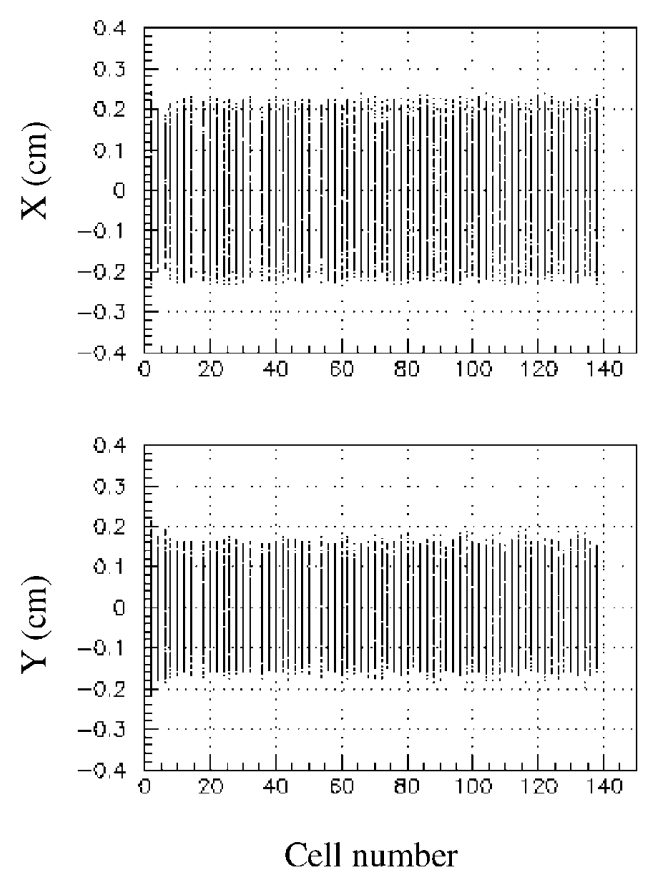

FIG. 8. (Color) Particle distributions in the transverse planes along the RFQ. 


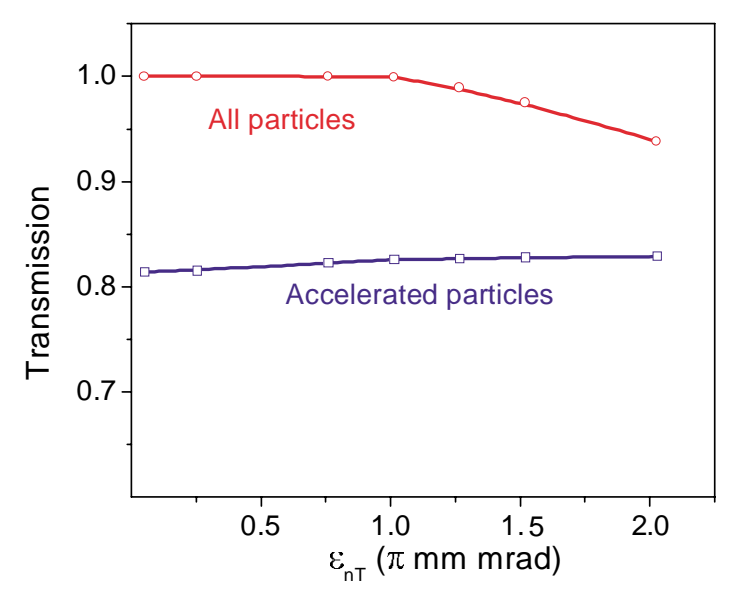

FIG. 9. (Color) Transmission as a function of the input transverse emittance. The red curve represents all particles at the end of the RFQ.

The longitudinal phase space plots of the two-chargestate uranium beam exiting the RFQ is shown in Fig. 6. For clarity of the analysis the longitudinal space plots of both charge states are shown with respect to the position of an average charge state $q=28.5$. In reality, different charge states will populate different buckets [4]. The distribution shown in Fig. 6 has a dense core containing 85\% of the accelerated particles and a halo. For each beam component, the emittance containing $99.9 \%$ of the accelerated particles is larger than the rms emittance by a factor of 10. Numerical simulations are consistent with the analytical approximations (3) (see also Fig. 2). It is clear that the longitudinal emittance is a function of the transverse emittance (see Fig. 7). The RFQ perfectly separates the accelerated and unaccelerated particles by their energies. The unaccelerated particles will be lost in the medium energy beam transport (MEBT). Since the beam energy in the MEBT is low, a negligible heat load due to the lost particles is expected .

The effective longitudinal emittance containing two charge states, $\mathrm{U}^{+28}$ and $\mathrm{U}^{+29}$, is bigger than the emittance for individual charge states due to the slightly different synchronous phases of each charge state. Despite an essential halo formation, the total longitudinal emittance of a two-charge-state beam is much lower than the acceptance of the following superconducting linac [7]. Moreover, we have found that only the external multiharmonic bunching and the design discussed above provide the lowest possible longitudinal emittance. The total effective emittance of the two-charge-state beam does not exceed $2.0 \pi \mathrm{keV} / \mathrm{u}$ nsec. The beam dynamics studies in the following superconducting linac show excellent beam properties for a multiple charge state uranium beam [7].

The RFQ is designed for the reference charge-to-mass ratio $q / \mathrm{A}=28.5 / 238$. The acceleration of charge states 28 or 29 produces a slight mismatched motion in the transverse phase space. Figure 8 shows transverse profiles of the single-charge state ${ }^{238} \mathrm{U}^{+28}$ beam along the RFQ where we observe some modulation of beam envelopes. The envelope $r_{\max , 28}$ is shown for accelerated particles only. The envelope modulation is determined by the expression

$$
\xi=\sqrt{\frac{r_{\max , 28}^{2}}{\rho_{\max , 28.5}^{2}} \frac{1}{\lambda \varepsilon_{n}}}
$$

and does not exceed 5\% for each charge state. There is no transverse emittance growth both for single- and twocharge-state beams along the RFQ. Figure 9 shows the transmission quality factors, calculated as a ratio of the number of particles at the end of the RFQ and the number of particles before the multiharmonic buncher. A small increase in the number of accelerated particles as the input beam emittance increases is related to the larger stability area for off axis particles.

The RFQ is followed by the MEBT which must match a two-charge-state beam to the following superconducting radio frequency linac. Our studies have shown [4] that superconducting solenoids are the best option for the MEBT. Beam waists in both transverse planes at the RFQ exit are preferable for matching to the following axial symmetric focusing channel. The last cell of the RFQ is designed in order to produce these desired beam waists (see Fig. 10).

Both ends of the RFQ structure considered here have a longitudinal voltage of $\sim 20 \mathrm{kV}$ on the axis as is inherent
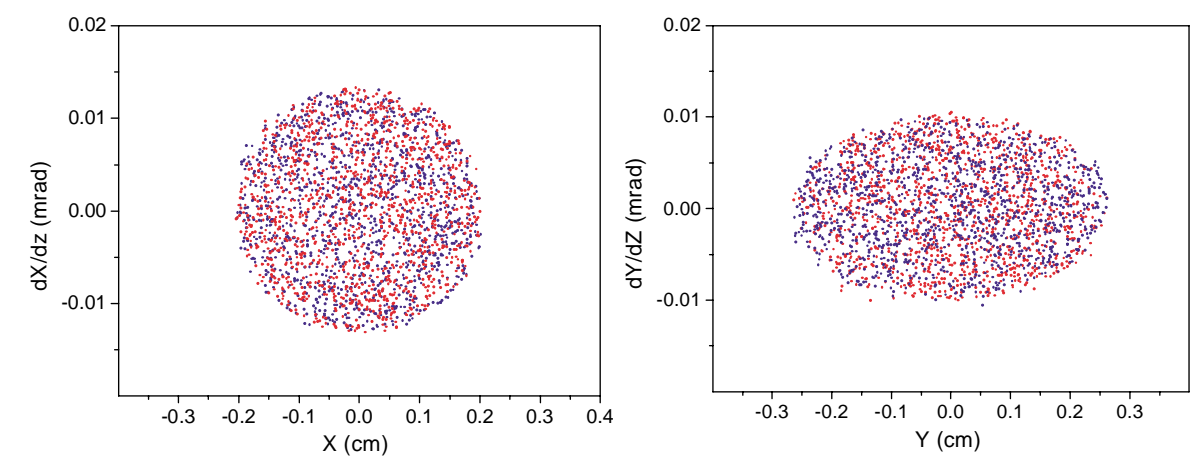

FIG. 10. (Color) Transverse phase space plots of ${ }^{238} \mathrm{U}^{+28}$ (red dots) and ${ }^{238} \mathrm{U}^{+29}$ (blue dots) beams exiting the RFQ. 
in split-coaxial structures. Beam dynamics simulations show that this voltage does not affect the beam parameters. At the low energy end the effect is negligible due to the long radial matching section. At the exit of the RFQ the effect is also small due to the relatively high energy of the particles.

\section{RFQ CAVITY DESIGN}

A conventional four-vane RFQ cavity with a longitudinal direction of the magnetic flux in the resonator chambers provides high shunt impedance due to the simple shape, and wide smooth paths for rf currents. Shunt impedances of most operating four-vane RFQs are close to shunt impedances obtained from three-dimensional electrodynamics codes. However, the four-vane structure is not appropriate in low frequency range due to the large transverse dimensions. In addition, special care must be taken in order to properly separate nonoperating modes. A cavity operating in a split-coaxial mode has been proposed and built for RFQs in the $\sim 12-25 \mathrm{MHz}$ frequency range. This type of resonant cavity reduces the transverse dimensions of the cavity significantly compared to the four-vane structure. In addition, the split-coaxial mode provides high stability of the accelerating field because the transverse magnetic field encircles all four vanes as in a coaxial waveguide.
Operational experience with a prototype $12 \mathrm{MHz}$ RFQ [8] does not show any multipacting in the full power range of zero to the designed value. This feature is very important for RFQs responsible for acceleration of various ion beam species.

The selection of the proper RFQ cavity operating at $57 \mathrm{MHz}$ in cw regime is still a challenge. For the best performance of the beam dynamics of two-charge-state beams it is highly desirable to select a symmetric resonant structure in order to avoid any possible dipole component of the electric and magnetic fields on the axis. Concerning fabrication, the furnace brazing technique has proven most reliable for cw RFQs. Several high-duty cycle RFQs were built on the base of a four-rod structure. However, these structures are intrinsically asymmetric. The dipole components of both electric and magnetic fields are present on the axis of the structure. In addition, a noticeable part of rf power losses occurs on the electrode's surface. This requires a complicated cooling system and can cause field distortions [9]. Another example of a low-frequency cw RFQ is the TRIUMF 35-MHz RFQ [10], which is a split-ring four-rod resonant structure. We found that the fabrication technique for this type of structure becomes much more complicated at higher frequency. On the basis of our previous experience in developing low-frequency RFQ cavities [11-14], we propose a new

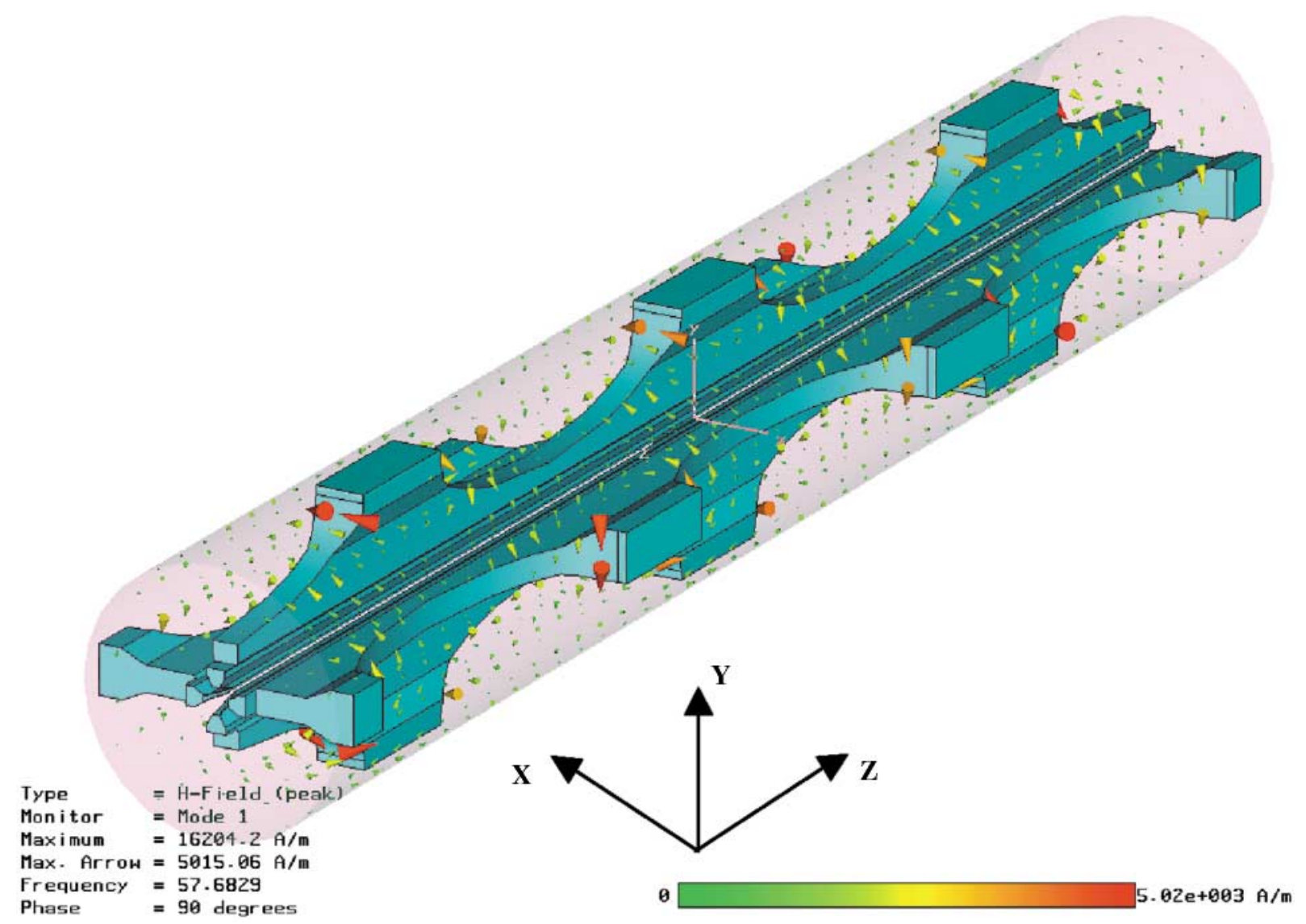

FIG. 11. (Color) Full length model of the RFQ cavity designed using the Mws code. The arrows show the magnetic field directions. 


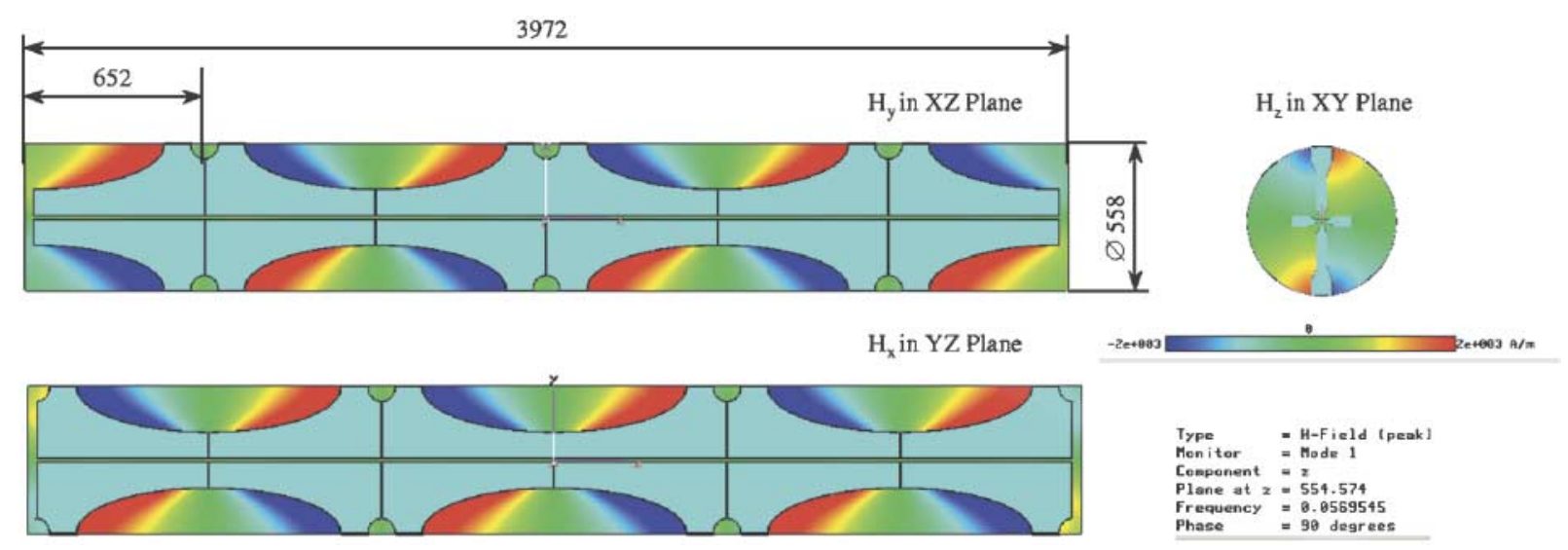

FIG. 12. (Color) Magnetic field density distribution in $X Z, Y Z, X Y$ cross sections of the RFQ resonator.

type of RFQ structure that combines the advantages of the four-vane and split-coaxial structures. An isometric view of the full-length computer model of the cavity is shown in Fig. 11. It can be considered to be a hybrid of a conventional four-vane structure with coupling "windows" between the chambers and split-coaxial cavities. The specific magnetic field distribution makes it possible to provide a resonant frequency of $57.5 \mathrm{MHz}$ within a $60-\mathrm{cm}$ diameter tank. This structure provides high shunt impedance, relatively simple mechanical design, moderate diameter, and large frequency separation of the operating and nonoperating modes. There are both longitudinal and transverse components of the magnetic field with respect to the longitudinal axis. Some of the magnetic flux is directed around the axis while some magnetic flux surrounds the stems, stabilizing the field distribution in the operating mode. The elliptical shape of the windows provides a low density rf current on the vane surface and improves the longitudinal flatness of the voltage distribution compared to other low-frequency RFQ structures. In addition, this vane shape provides good mechanical stability and the possibility of a modular design of the RFQ cavity.

The final RFQ cavity is $558 \mathrm{~mm}$ in diameter and consists of six 652-mm long modules. The exact dimensions of the cavity and vanes are under optimization with respect to thermal and structural stability. The preliminary shape of the vanes and magnetic field distributions are shown in Fig. 12.

TABLE III. Calculated electrodynamic parameters of the cavity.

Frequency of operating mode

Frequency of the nearest mode

Frequency of the second nearest mode

$Q$ factor

Total rf power losses

Specific rf power losses
$57.5 \mathrm{MHz}$

$68.4 \mathrm{MHz}$

$93.5 \mathrm{MHz}$

12000

$45 \mathrm{~kW}$

$11.5 \mathrm{~kW} / \mathrm{m}$
Electrodynamics simulations of the cavity have been carried out using the CST Micro-Wave Studio (Mws) [15] and OPERA 3D codes. The main results are presented in Table III. Radio frequency power losses were calculated taking into account realistic distributions of electrical field between the vanes. The focusing gradient simulated by the Mws code is presented in Fig. 13. The losses have been determined for a design gradient value $G_{0}=U_{0} / R_{0}^{2}=$ $190 \mathrm{kV} / \mathrm{cm}^{2}$. The use of vane-to-vane voltage for the calculation of rf power losses can lead to underestimation of these losses.

The results of the electrodynamics calculations are used for further finite element analysis of the thermal and mechanical properties of the cavity. Radio frequency voltage variation along the vanes is an inevitable feature of any longitudinally nonuniform structure. In our case, the magnitude of the voltage variation depends mostly on the longitudinal size of the window: the longer the section at a given diameter, the larger the voltage variation. At the same time, the elliptical shape of the window reduces the field

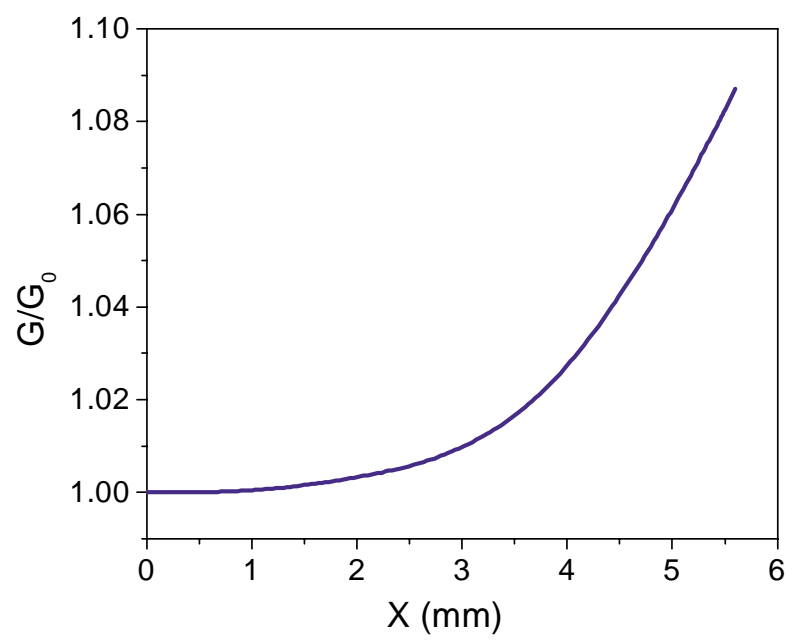

FIG. 13. (Color) Gradient of the electric field as a function of transverse distance. 


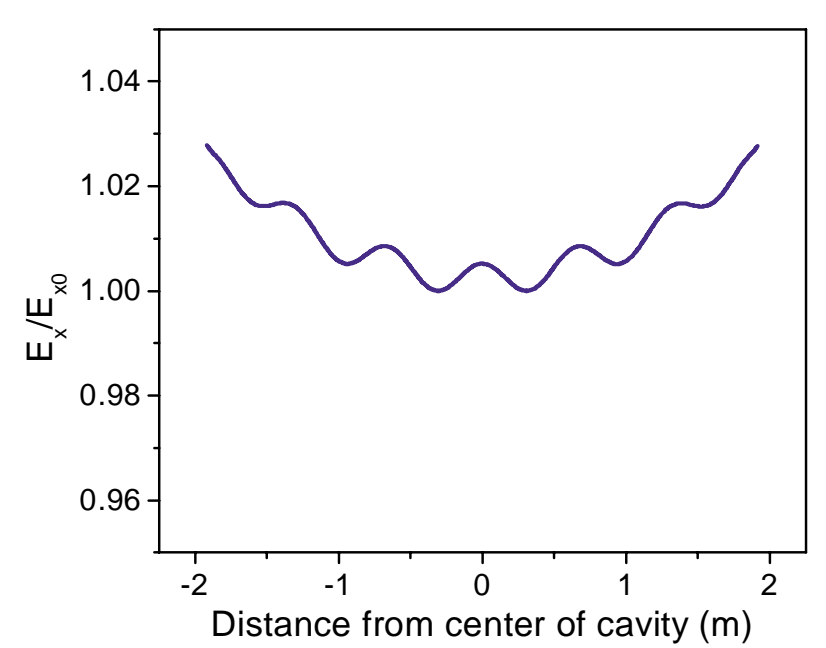

FIG. 14. Transverse electric field along the RFQ with unmodulated vanes.

variation, compared to a rectangular window. The highest voltage deviation occurs at the ends of the vanes where an additional capacitance between the vanes and the end plate exists. Figure 14 shows a distribution of the transverse component of the electric field along the cavity. The spacing between the vane edge and the end plate is 30 $\mathrm{mm}$. The maximum deviation of the field along the cavity is $\pm 1.3 \%$. The voltage variation within a module is $\pm 0.23 \%$. The radial matching section of the RFQ can also perturb the voltage flatness. However, a final uniform field distribution along the RFQ may be obtained using standard water-cooled slug tuners.

One of the main parameters of any RFQ cavity is the sensitivity of the rf field distribution to misalignment of the vanes. In order to estimate the influence of the electrode misalignment introduced by an asymmetry in the cavity, simulations with the 3D OPERA have been carried out. The following relations were found for voltage and frequency deviations as a function of radial displacement of the vane,

$$
\frac{\Delta U}{U}[\%]=-6.7 \Delta r[\mathrm{~mm}], \quad \frac{\Delta f}{f}=0.009 \Delta r[\mathrm{~mm}] .
$$

The voltage and frequency deviations can be controlled by requiring reasonable tolerances of the vane displacement to less than $\pm 100 \mu \mathrm{m}$.

\section{CONCLUSIONS}

A $57.5 \mathrm{MHz}$ cw RFQ for the RIA driver linac has been designed to ensure stable cw operation and formation of a high-quality beam containing two charge states. The beam dynamics have been optimized to obtain the specified twocharge-state beam parameters while maintaining relatively low peak electric field on the vane surface (1.25 Kilpatrick units). The RFQ maintains low longitudinal emittance of the two-charge-state beam formed by the multiharmonic buncher. There is no transverse emittance growth of the two-charge-state beam along the RFQ.

A new resonant structure has been developed for the RFQ. It combines advantages of the conventional four-vane and split-coaxial structures. Three-dimensional electrodynamics simulations confirmed that this RFQ structure has high shunt impedance and large frequency separation of the modes.

Fabrication of both a low power "cold model" of the complete RFQ and a single segment "engineering model" is planned for the near future.

\section{ACKNOWLEDGMENT}

This work is supported by the U.S. Department of Energy, Nuclear Physics Division, under Contract No. W-31109-ENG-38.

[1] K. W. Shepard, J. R. Delayen, C. M. Lyneis, J. A. Nolen, P. N. Ostroumov, J. W. Staples, J. Brawley, C. Hovater, M. Kedzie, M. P. Kelly, J. Mammosser, C. Piller, and M. Portillo, in Proceedings of the 9th International Workshop on RF Superconductivity, Santa Fe, New Mexico, 1999, edited by B. Rusnak (LANL, Los Alamos, 2000), p. 345.

[2] A. A. Kolomiets and S.G. Yaramishev, Institute of Theroretical and Experimental Physics internal report, 1998 (unpublished).

[3] A. I. Balabin, Institute of Theroretical and Experimental Physics Report No. ITEP-107, 1981.

[4] P. N. Ostroumov, K. W. Shepard, V. N. Aseev, and A. A. Kolomiets, in Proceedings of the 2000 Linac Conference, Monterey, California, Report No. SLAC-R-561, 2000, edited by A. W. Chao, p. 202.

[5] I. M. Kapchinskiy, Theory of Linear Resonant Accelerators (Energoizdat, Moscow, 1982) (in Russian).

[6] A. A. Kolomiets, V. Pershin, I. Vorobyov, S. Yaramishev, and Ju. Klabunde, in Proceedings of the Sixth European Particle Accelerator Conference, Stockholm, Sweden, 1998, edited by S. Myers et al. (IOP, Bristol, UK, 1998), p. 1201.

[7] P. N. Ostroumov, Phys. Rev. ST. Accel. Beams 5, 030101 (2002).

[8] R. A. Kaye, K. W. Shepard, B. Clifft, and M. Kedzie, in Proceedings of the 1999 IEEE Particle Accelerator Conference, New York, 1999, edited by A. Luccio and W. MacKay (IEEE, Piscataway, NJ, 1999), p. 524.

[9] H. Vormann, A. Schempp, U. Bessler, C. W. Planner, and A. Letchford, in Proceedings of the Sixth European Particle Accelerator Conference, Stockholm, Sweden, 1998 (Ref. [6]), p. 782.

[10] R. L. Poirier, R. Baartman, P. Bricault, K. Fong, S. Koscielniak, R. Laxdal, A. K. Mitra, L. Root, G. Stanford, and D. Pearce, in Proceedings of the 2000 Linac Conference, Monterey, California (Ref. [4]), p. 1023.

[11] V. A. Andreev and G. Parisi, in Proceedings of the Particle Accelerator Conference, Washington, DC, 1993 (IEEE, Piscataway, NJ, 1993), p. 3124.

[12] V.A. Andreev, A. Kolomiets, S. Yaramishev, and J. Klabunde, in Proceedings of Linac'94, Tsukuba, Japan, 
1994, edited by K. Takata (KEK, Tsukuba, Japan, 1994), p. 713.

[13] V.A. Andreev, A.A. Kolomiets, V.I. Pershin, V.N. Sidorenko, R. M. Vengrov, S. G. Yaramyshev, O. V. Ershov, and G. Parisi, in Proceedings of the International Particle Accelerator Conference, Vancouver, Canada, 1997, edited by M. Comyn et al. (IEEE, Piscataway, NJ, 1998), p. 1090.
[14] D. Kashinsky, A. Kolomiets, T. Kulevoy, R. Kuybida, V. Kuzmichov, S. Minaev, V. Pershin, B. Sharkov, R. Vengrov, and S. Yaramishev, in Proceedings of the Seventh European Particle Accelerator Conference, Vienna, 2000, http://accelconf.web.cern.ch/AccelConf/e00/index.html, p. 854.

[15] CST Microwave Studio, User Manual Version 3.0, 2001, CST GmbH, Darmstadt, Germany, http://www/cst/de/. 